\title{
Arginase-II promotes melanoma migration and adhesion through enhancing hydrogen peroxide production and STAT3 signaling
}

\author{
Yi Yü ${ }^{1,2}$ (1) | Diogo Ladeiras ${ }^{1}$ | Yuyan Xiong ${ }^{1,2}$ (D) Kayluz Frias Boligan ${ }^{3}$ | \\ Xiujie Liang $^{1}$ | Stephan von Gunten ${ }^{3}$ | Robert E. Hunger ${ }^{4}$ | Xiu-Fen Ming ${ }^{1}$ (D) | \\ Zhihong Yang ${ }^{1}$ (D)
}

\begin{abstract}
${ }^{1}$ Laboratory of Cardiovascular and Aging Research, Department of Endocrinology, Faculty of Science and Medicine, Medicine Section, Metabolism and Cardiovascular Medicine, University of Fribourg, Fribourg, Switzerland

${ }^{2}$ Key Laboratory of Resource Biology and Biotechnology in Western China, Ministry of Education, School of Medicine, Northwest University, Xi'an, Shaanxi, China

${ }^{3}$ Institute of Pharmacology, University of Bern, Bern, Switzerland

${ }^{4}$ Department of Dermatology, Bern University Hospital Inselspital, University of Bern, Bern, Switzerland

\section{Correspondence}

Xiu-Fen Ming and Zhihong Yang, Laboratory of Cardiovascular and Aging Research, Faculty of Science and Medicine, Medicine Section,

Department of Endocrinology, Metabolism and Cardiovascular Medicine, University of Fribourg, 1700 Fribourg, Switzerland. Email: xiu-fen.ming@unifr.ch (X.-F. M.) and zhihong.yang@unifr.ch (Z. Y.)
\end{abstract}

\section{Present address}

Key Laboratory of Resource Biology and Biotechnology in Western China, Ministry of Education, School of Medicine, Northwest University, Xi'an, Shaanxi, China.

\section{Funding information}

Swiss Heart Foundation (SHF); Swiss National Science Foundation (SNF),

Grant/Award Numbers: 31001A_179261/1, 310030162552,310030184757,

31003A_159582/1; Swiss Cancer League/ Swiss Cancer Research, Grant/Award Number: KFS-3941-08-2016

\begin{abstract}
Elevated arginase type II (Arg-II) associates with higher grade tumors. Its function and underlying molecular mechanisms in melanoma remain elusive. In the present study, we observed a significantly higher frequency of Arg-Il expression in melanoma of patients with metastasis than those without metastasis. Silencing Arg-II in two human melanoma cell lines slowed down the cell growth, while overexpression of native but not a catalytically inactive Arg-Il promoted cell proliferation without affecting cell death. Treatment of cells with arginase inhibitor also reduced melanoma cell number, demonstrating that Arg-II promotes melanoma cell proliferation dependently of its enzymatic activity. However, results from silencing Arg-II or overexpressing native or the inactive Arg-II as well as treatment with arginase inhibitor showed that Arg-II promotes melanoma metastasis-related processes, such as melanoma cell migration and adhesion on endothelial cells, independently of its enzymatic activity. Moreover, the treatment of the cells with STAT3 inhibitor suppressed Arg-II-promoted melanoma cell migration and adhesion. Furthermore, catalase, but not superoxide dismutase, prevented STAT3 activation as well as increased melanoma cell migration and adhesion induced by overexpressing native or the inactive Arg-II. Taken together, our study uncovers both activity-dependent and independent mechanisms of Arg-II in promoting melanoma progression. While Arg-II enhances melanoma cell proliferation through polyamine dependently of its enzymatic activity, it promotes metastasis-related processes, that is, migration and adhesion onto endothelial cell, through mitochondrial $\mathrm{H}_{2} \mathrm{O}_{2}$-STAT3 pathway independently of the enzymatic activity. Suppressing Arg-II expression rather than inhibiting its enzymatic activity may, therefore, represent a novel strategy for the treatment of melanoma.
\end{abstract}

KEYWORDS

arginase-II, melanoma, metastasis, ROS, STAT3 


\section{1 | INTRODUCTION}

Melanoma is an aggressive type of skin cancer, and widespread metastasis is the main cause of death in patients with melanoma. In general, the process of tumor metastasis involves a series of stages including detachment from the primary tumor, invasion and migration, intravasation, survival in the blood circulation and adhesion to endothelial cells, extravasation, and finally proliferation in distant organs (Wirtz, Konstantopoulos, \& Searson, 2011). The ability of migration and adhesion of circulating tumor cells to endothelial cells is crucial for this process (Tang \& Honn, 1994). Multiple molecular mechanisms are involved in control of metastasis. One of the most recognized signal transduction pathways in cancer metastasis is the signal transducer and activator of transcription 3 (STAT3) protein. Increasing evidence has demonstrated the constitutive activation of STAT3 in a wide variety of human tumors (Siveen et al., 2014). The activated STAT3 protein has been found to associate with metastasis in various organs, and inhibition of STAT3 exerts antitumor and antimetastasis effects (Kamran, Patil, \& Gude, 2013). The target genes of STAT3 are involved in multiple steps of tumor initiation and metastasis, including cell survival through inhibition of p53 (Niu et al., 2005), invasion and migration by upregulating matrix metalloproteinases and Rho-GTPase (Debidda, Wang, Zang, Poli, \& Zheng, 2005; Dechow et al., 2004; Xie et al., 2004), and angiogenesis by vascular endothelial growth factor activation (Schaefer, Ren, Fuller, \& Schaefer, 2002).

The type II I-arginine:ureahydrolase, arginase-II (Arg-II), is a mitochondrial enzyme that is inducible and widely expressed in many extrahepatic tissues/cells. The functions are attributed to the hydrolysis of L-arginine to urea and L-ornithine, the precursor for the biosynthesis of the polyamines, which is involved in cell growth and protein biosynthesis (Kusano, Berberich, Tateda, \& Takahashi, 2008) Elevated arginase activity and expression, especially Arg-Il but not Arg-I expression, have been detected in various cancer tissues and cells such as prostate, breast, lung, colon, and pancreatic cancers (Bronte et al., 2005; Gokmen, Aygit, Ayhan, Yorulmaz, \& Gulen, 2001; Porembska et al., 2003; Porembska, Zabek, Grabon, Rahden-Staron, \& Baranczyk-Kuzma, 2001; Rotondo et al., 2008; Zaytouni et al., 2017) Moreover, it is also reported that high Arg-Il expression was associated with high-grade tumors and predicts poor outcome (Gokmen et al., 2001; Ino et al., 2013; Rotondo et al., 2008), and that the inhibition of arginase suppresses cancer cell growth and metastasis (Secondini et al., 2017; Singh et al., 2013; Tate et al., 2008; Zaytouni et al., 2017). Moreover, it has also been suggested that excessive activity of arginase is associated with immune suppression by depleting arginine from immune cells resulting in inhibition of $\mathrm{T}$ cell activation and proliferation in the tumor microenvironment (Avtandilyan, Javrushyan, Petrosyan, \& Trchounian, 2018; Bronte et al., 2005; Setty et al., 2016; Tate et al., 2008; Timosenko, Hadjinicolaou, \& Cerundolo, 2017). Although high correlation between Arg-II and cancer was found in the clinic, the exact function and the underlying mechanisms of Arg-II in solid tumor have not been fully elucidated. The aim of this study is to investigate whether and how Arg-II is involved in human melanoma cell growth and metastasis in patients' samples and melanoma cell lines.

\section{I MATERIALS AND METHODS}

\section{1 | Materials}

Reagents were purchased or obtained from the following sources: rabbit antibody against Arg-II (sc-20151), and mouse antibody against ICAM-1(sc-8439), STAT3 (sc-8019) were from Santa Cruz Technology Inc. (Nunningen, Switzerland); rabbit antibody against phospho-STAT3-Tyr705 (9145) was purchased from Cell Signaling (Allschwil, Switzerland); PhosphoDetect ${ }^{\mathrm{TM}}$ mouse anti-p66Shc (p66Shc-Ser36; \#566807) was purchased from Millipore (Zug, Switzerland). Rabbit anti-p66Shc (\#06-203) was from Upstate (Lucerna Chem, Lucerne, Switzerland); mouse antibody against tubulin (T9026) was from Sigma (Buchs, Switzerland); mouse antibody against Melan-A (M719629-2) was from DAKO (Agilent Technologies, Basel, Switzerland). IRDye 800 conjugated affinity-purified goat anti-rabbit immunoglobulin G (IgG) F(c) (926-32211) was purchased from BioConcept (Allschwil, Switzerland), Alexa fluor 680 conjugated goat anti-mouse IgG $(\mathrm{H}+\mathrm{L}$; (A21057), goat anti-mouse $\operatorname{lgG}(\mathrm{H}+\mathrm{L})$ secondary antibody Alexa Fluor ${ }^{\circledR} 594$ conjugate (A32742), goat antirabbit IgG $(\mathrm{H}+\mathrm{L})$ secondary Antibody Alexa Fluor ${ }^{\circledR} 488$ conjugate (A32731), and Hoechst 33342 were from Invitrogen/Thermo Fisher Scientific (Waltham, MA). Stattic (S7947) was from Sigma (Buchs, Switzerland). Alpha-difluoromethylornithine (DFMO; 288500) was from Merck KGaA (Darmstadt, Germany). S-(2-boronoethyl)L-cysteine (BEC; 197900) was from Calbiochem (VWR, Geneva, Switzerland). All cell culture media and materials were purchased from Gibco BRL (Lucerne, Switzerland).

\subsection{Generation of recombinant adenoviral constructs}

Generation of recombinant adenovirus ( $r A d$ ) expressing short hairpin RNA (shRNA) targeting human Arg-II driven by the U6 promoter ( $\mathrm{rAd} / \mathrm{U} 6-A R G-I^{\text {shRNA}}$ ) and its control rAd/U6-LacZ ${ }^{\text {shRNA }}$ as well as $\mathrm{rAd} / \mathrm{CMV}$ empty vector and $\mathrm{rAd} / \mathrm{CMV}-\mathrm{ARG}$-II, $\mathrm{rAd} / \mathrm{CMV}$ ARG-II-H160F (a catalytically inactive mutant of Arg-II), rAd/CMVCATALASE, rAd/CMV-SOD2 were generated as previously described (Yepuri et al., 2012).

\section{3 | Cell culture and adenoviral transduction}

Cultivation and transduction of human umbilical vein endothelial cells (HUVECs) were performed as described previously (Ming et al., 2002). ME276 and ME275, human melanoma cell lines derived from the lymph node of two different patients with melanoma metastasis (Caballero et al., 2010), were cultured with Roswell Park 
Memorial Institute (RPMI)-1640 containing 10\% fetal calf serum (FCS). Cells were transduced with the rAd at titers of $\sim 500$ multiplicities of infection and then cultured in complete medium for 2-4 days before experiments.

\subsection{Cell number counting}

Growth of ME276 and ME275 cells was assessed by cell number, which was counted with the TC20 automated cell counter (BIO-RAD).

\section{5 | Tumor adhesion assay}

For tumor adhesion assay, $2 \times 10^{5}$ melanoma cells were washed twice with phosphate-buffered saline (PBS) and then labeled with $5 \mu \mathrm{mol} / \mathrm{L} \mathrm{CFDA-SE}$ in PBS at $37^{\circ} \mathrm{C}$ for $8 \mathrm{~min}$. The labeling was stopped by adding $1 \mathrm{ml}$ of FCS for $1 \mathrm{~min}$. The labeled melanoma cells were washed twice with PBS, suspended in RPMI-1640 medium containing $10 \% \mathrm{FCS}$, and then added to confluent HUVECs layers in 12 wellplates. After incubation for $20 \mathrm{~min}$ at $37^{\circ} \mathrm{C}$, the nonadherent melanoma cells were washed away by PBS and the adherent melanoma cells were fixed with $3.7 \%$ paraformaldehyde. The images of adherent melanoma cells were captured under a fluorescent microscope. The number of adherent melanoma cells was counted using Image-J software.

\subsection{Wound healing assay}

Migration of melanoma cells was measured by wound healing assay. Ninety percent of confluent melanoma cell layers were wounded by scratching with a sterile $200 \mu$ l pipette tip. Then, the cells continued to be cultured in serum-free medium (RPMI-1640 medium containing $0.2 \% \mathrm{FCS}$ ) at $37^{\circ} \mathrm{C}$ for $24 \mathrm{hr}$. A digital camera system was used to acquire images of the scratches of the cells at 0 and $24 \mathrm{hr}$. Wound surface area was quantified by image analysis (Image-J).

\section{7 | Immunoblotting}

Cell lysate preparation, sodium dodecyl sulfate-polyacrylamide gel electrophoresis (SDS-PAGE), transfer of SDS gels to an Immobilon-P membrane (Millipore) were performed as previously described (Yepuri et al., 2012). The resultant membrane was first incubated with the corresponding primary antibody at $4^{\circ} \mathrm{C}$ overnight with gentle agitation after blocking with $5 \%$ skimmed milk. The blot was then further incubated with a corresponding anti-mouse (Alexa fluor 680 conjugated) or anti-rabbit (IRDye 800 conjugated) secondary antibody. Signals were visualized using the Odyssey Infrared Imaging System (LI-COR Biosciences). Quantification of the signals was performed using NIH Image 1.62 software (U. S. National Institutes of Health).

\section{8 | Arginase activity assay}

Arginase activity in melanoma cell lysates was measured by colorimetric determination of urea formed from L-arginine in an in vitro activity assay, as previously described (Ming et al., 2004). Briefly, equal amount of the cell lysate was added to $50 \mu \mathrm{l}$ of Tris- $\mathrm{HCl}$ (10 mmol/L pH 7.4) containing $5 \mathrm{mmol} / \mathrm{L} \mathrm{MnCl}_{2}$. Arginase was then activated by heating the mixture at $56^{\circ} \mathrm{C}$ for $10 \mathrm{~min}$. Then, $100 \mu \mathrm{l}$ of L-arginine $(100 \mathrm{mmol} / \mathrm{L}[\mathrm{pH} 9.6])$ was added at $37^{\circ} \mathrm{C}$ for $1 \mathrm{hr}$ for hydrolysis reaction. For colorimetric determination of urea, $1 \mathrm{ml}$ of chromogenic reagent consisting of 1 volume of 3\% 2,3-butanedione monoxide and 29 volumes of the acid solution mixture $\left(\mathrm{H}_{2} \mathrm{SO}_{4}\right.$ : $\mathrm{H}_{2} \mathrm{PO}_{4}: \mathrm{H}_{2} \mathrm{O}$ 1:3:7) was added. The mixture was then heated at $100^{\circ} \mathrm{C}$ for $30 \mathrm{~min}$. After placing the samples in the dark for $30 \mathrm{~min}$ at room temperature, the urea concentration was determined spectrophotometrically by the absorbance at $492 \mathrm{~nm}$.

\section{9 | Mitochondrial superoxide and hydrogen peroxide detection}

Mitochondrial superoxide was detected by using MitoSOX ${ }^{\mathrm{TM}}$ Red Mitochondrial Superoxide Indicator (Invitrogen, M36008) followed by nuclei counterstaining with Hoechst 33342. The immunofluorescence signals were visualized under LEICA TCS SP5 Confocal Microscope. Mitochondrial hydrogen peroxide was detected by costaining of mitochondria and hydrogen peroxide using MitoTracker (M22426, Thermo Fisher Scientific) and cell-based Hydrogen Peroxide Assay Kit (Abcam, ab138874), respectively, according to the manufacturers' instructions, respectively. The immunofluorescence signals were visualized under Zeiss fluorescence microscope.

\subsection{0 | Cell death assay}

The melanoma cell death was detected with an Annexin-V-FLUOS Staining Kit (\#1988549, Roche Applied Science) following the manufacturer's instruction. The signals were evaluated by fluorescence microscopy, and the green signal from Annexin-V-FLUOS indicates apoptotic cells while the red signal from propidium iodide indicates necrotic cells.

\subsection{1 | Immunofluorescence staining of human melanoma tissue}

Archived tissue samples of primary and metastatic melanoma from patients at the Department of Dermatology of Inselspital (Bern, 
Switzerland) were included in this study. All studies using human material were in accordance with the guidelines of, and approved by, the cantonal ethical committees of Bern.

For the immunostaining analysis, paraffin-embedded tissue sections of $6 \mu \mathrm{m}$ were deparaffinized using NeoClear. After deparaffinization, hydration in ethanol, and antigen retrieval in TRIS-EDTA $\mathrm{pH} 9$, tissue sections were blocked with $5 \%$ goat serum in PBS for $1 \mathrm{hr}$ at room temperature and then were incubated with the primary antibodies against Arg-II and melan-A overnight at $4^{\circ} \mathrm{C}$. Subsequently, tissue sections were incubated with fluorescently labeled secondary antibodies for $2 \mathrm{hr}$ at room temperature, followed by nuclei counter-staining with $300 \mathrm{nmol} / \mathrm{L}$ 4',6-diamidino-2-phenylindole for $3 \mathrm{~min}$. Slides were mounted with ProLong ${ }^{\mathrm{TM}}$ Gold Antifade Mountant and immunofluorescence signals were scanned in a 3DHistech Pannoramic Scan (Sysmex). Quantification of Arg-II staining was analyzed using the Allred scoring system (Guo et al., 2019) which is composed of proportion score and intensity score. Proportion score is defined as 0, 1, 2, 3, 4, 5 representing 0 , $\leq 1 \%, 1-10 \%, 11-33 \%, 34-66 \%$, and $67-100 \%$ of cells positive, respectively. Intensity score is defined as 0, 1, 2, 3 representing negative, weak, intermediate, and strong signals, respectively.

\subsection{2 | Statistics}

Data are given as mean \pm standard error of the mean. In all experiments, $n$ indicates the number of individual experiments. Statistical analysis was performed with unpaired Student's $t$ test or analysis of variance with Dunnett or Bonferroni posttest. Differences in mean values were considered significant at $p<0.05$

\section{3 | RESULTS}

\section{1 | Arg-II expression is associated with melanoma with metastasis in patients}

Arginase, especially Arg-II, was reported to be detected in various cancer tissues and cells, but the study about Arg-II in melanoma was lacking. In our study, we examined the expression of Arg-II in skin samples from melanoma patients by coimmunofluorescence staining of Arg-Il and melanoma cells marker Melan-A, as shown in Figure 1a. By analyzing 13 skin samples from patients with primary melanoma and 14 skin samples from patients with metastatic melanoma, the expression of Arg-II is summarized in Figure 1b, which reveals that there is barely Arg-II expressed in skin melanoma cells from patients with primary melanoma, and only one sample showed weak Arg-II signal in a small portion (less than 10\%) of melanoma cells. However, over $50 \%$ of skin samples from patients with metastatic melanoma showed Arg-II expression in melanoma cells and three of them showed strong Arg-II expression and higher portion (more than 10\%) of Arg-II-positive melanoma cells. The significant difference of Arg-II expression between nonmetastatic and metastatic melanoma suggests that Arg-II expression is associated with metastatic spread.

\section{2 | Arg-II promotes the growth of melanoma cells through polyamine synthesis}

Fast proliferation and growth of tumor cells is the main feature of cancer and also the initiation step for tumor metastasis. To understand the function of Arg-II in cancer cell growth, human melanoma ME276 cells were used and Arg-II was silenced by shRNA in the cells. As shown in Figure 2a, the melanoma cells express high levels of Arg-II (Arg-I was not detectable) and silencing Arg-II significantly reduced the cell number as compared to the control cells. Treatment of the cells with arginase inhibitor BEC achieved the similar effect (Figure 2b). In line with these data, the higher cell number was found in cells overexpressing the native Arg-II while the cells overexpressing a catalytically inactive mutant of Arg-II (H160F) had comparable cell number to control cells (Figure 2c). Similar results were obtained in another melanoma cell line ME275 (Figure S1). Moreover, treatment of the cells with DFMO that inhibits polyamine biosynthesis from L-ornithine, the product of arginase, reverted Arg-II overexpression-induced increase in cell number (Figure 2c). Cell death assay assessing both necrotic and apoptosis cell number showed that Arg-II had no effect on melanoma cell death (Figure S2). These data indicate that Arg-II promotes the human melanoma cell growth through polyamine synthesis in an enzyme activity-dependent manner.

\section{3 | Arg-II promotes melanoma cell migration and adhesion on endothelial cells independently of its enzymatic activity}

Migration is a crucial stage for tumor cells to acquire migratory characteristics and invade through the extracellular matrix and basement membrane, while the adhesion of tumor cells to vascular endothelial cells is a vital process for tumor extravasation. To evaluate the effects of Arg-II on the migration and the adhesion of melanoma cells to endothelial cells, a wound-healing assay and adhesion assay were performed, respectively. The human melanoma cells ME276 with Arg-II-silencing displayed slower migration rate (Figure 3a) and reduced adhesion onto endothelial cells (Figure 3b). Similar results were obtained in another melanoma cell line ME275 (Figure S3). However, treatment of the cells with arginase inhibitor BEC did not affect melanoma cell migration (Figure 3c) nor adhesion (Figure $3 d$ ). Consistent with these results, higher migration activity (Figure 3e) and more adherent melanoma cells (Figure 3f) were observed in cells overexpressing both native Arg-II and the inactive mutant of Arg-II H160F, which was verified in ME275 (Figure S4). This finding indicates an important role of Arg-II in the migration and adhesion of melanoma cells, which is independent of its enzymatic activity. 
(a)
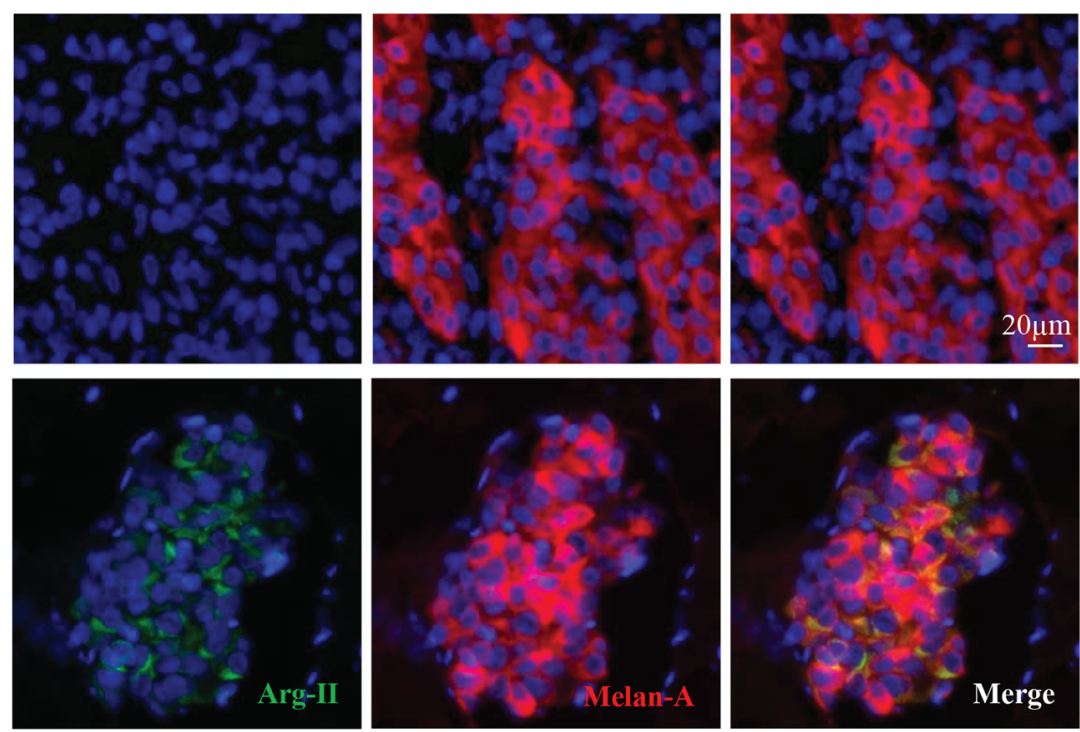

Skin tissue

from patient with primary melanoma
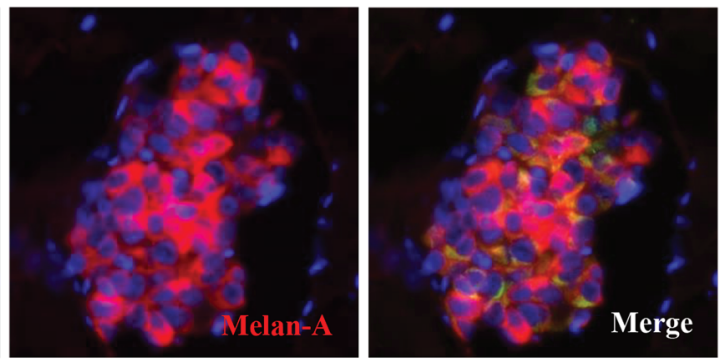

Skin tissue from patient with metastatic melanoma

(b)

Expression of Arg-II in patients with melanoma

\begin{tabular}{|c|c|c|c|}
\hline \multicolumn{2}{|c|}{ Melanoma samples } & $\begin{array}{c}\text { Primary } \\
(\mathrm{n}=13)\end{array}$ & $\begin{array}{c}\text { Metastatic } \\
(\mathrm{n}=14)\end{array}$ \\
\hline \multirow{4}{*}{ Arg-II expression } & - & 12 & 5 \\
\cline { 2 - 4 } & $-/+$ & 0 & 2 \\
\cline { 2 - 4 } & $\leq 10 \%$ & 1 & 4 \\
\cline { 2 - 4 } & $\geq 10 \%$ & 0 & 3 \\
\hline
\end{tabular}

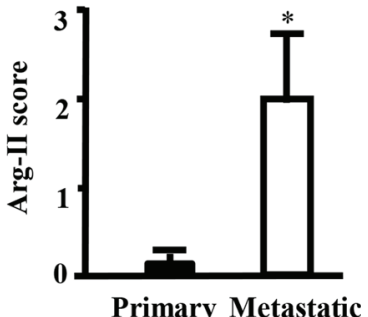

- : negative; -/+ : undefined

$\%$ : ratio of Arg-II and Melan-A double-positive area to Melan-A-positive area

FIGURE 1 Arg-II expression is associated with metastatic melanoma. (a) Immunofluorescence staining of human melanoma tissue. The skin tissue from patient with primary melanoma (upper panel) or metastatic melanoma (lower panel) were coimmunostained for Melan-A (red) and Arg-II (green) followed by counterstaining with DAPI (blue). The merged images are also shown. (b) A summary of Arg-II expression in patients with melanoma. Quantification of Arg-II staining using the Allred scoring system is shown in the bar graphs. ${ }^{*} p<.05$ versus primary group. Arg-II, arginase type II; rAd, recombinant adenovirus

\section{4 | Silencing Arg-II reduces mitochondrial superoxide anion and hydrogen peroxide $\left(\mathrm{H}_{2} \mathrm{O}_{2}\right)$ production and STAT3 signaling pathway in melanoma cells}

The effect of Arg-II on migration and adhesion of melanoma cells suggests its potential role in melanoma metastasis, mechanisms how Arg-II regulates this process is further investigated. The role of aberrant STAT3 activity in promoting tumor growth and metastasis was analyzed. Immunoblotting results in Figure 4a showed that phosphorylation of STAT3, but not total STAT3, was significantly decreased in cells with Arg-II-silencing. Although nuclear factor- $\mathrm{kB}$ and $\mathrm{mTORC} 1 / \mathrm{S} 6 \mathrm{~K} 1$ signaling were also well known being involved in tumor metastasis process, silencing Arg-II in ME276 cells had no effect on these signaling pathways (Figure S5). In addition, the expression level of ICAM-1, which was reported to be detected in numerous cancer types and linked with tumor development and progression, was also significantly reduced by silencing Arg-II (Figure 4a). These results were further confirmed in another melanoma cell line ME275 (Figure S6a). In line with the activity-independent effects of Arg-II on melanoma cell migration and adhesion onto endothelial cells, treatment of ME276 with the arginase inhibitor BEC did not affect protein levels of p-STAT3 or ICAM-1 (Figure 4b).

Reactive oxygen species (ROS), such as superoxide anion, $\mathrm{H}_{2} \mathrm{O}_{2}$, and hydroxyl radical, are well-known regulators in various metastatic processes, such as adhesion, invasion, and proliferation (Belkhiri, Richards, Whaley, McQueen, \& Orr, 1997). Our previous study showed that Arg-II causes mitochondrial dysfunction and induces mitochondrial ROS production in smooth muscle cells (Xiong, Yu, Montani, Yang, \& Ming, 2013). We thus examined the effect of Arg-II on mitochondrial ROS production in melanoma cells. The results revealed that silencing Arg-II in ME276 cells inhibited mitochondrial superoxide anion production, as shown in 
(a)

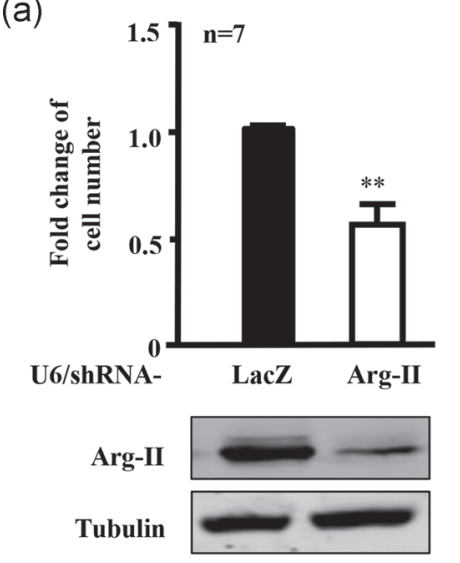

(c)

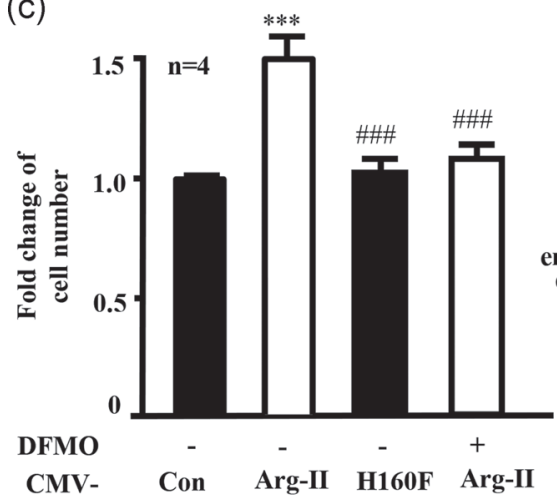

(d)

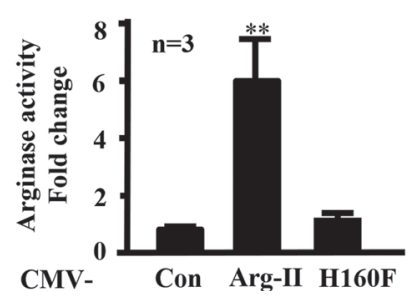

(b)

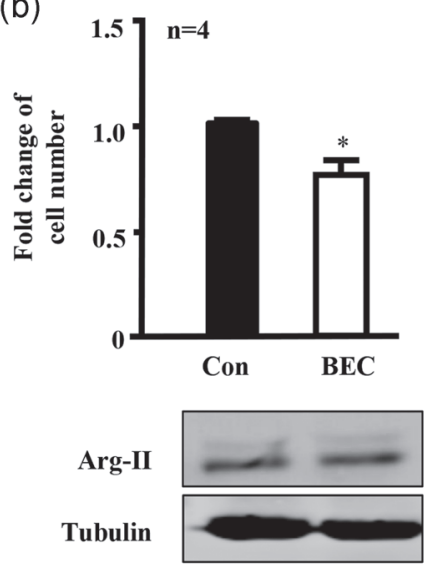

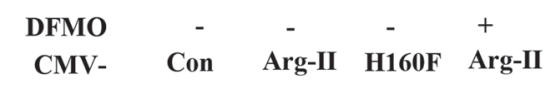
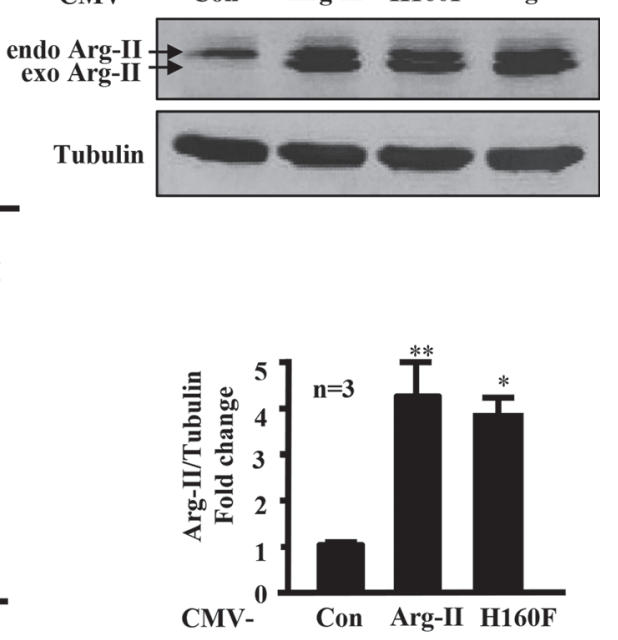

FIGURE 2 Arg-II promotes the growth of melanoma cells through polyamine synthesis. (a) ME276 cells were transduced with rAd/U6LacZ $^{\text {shRNA }}$ as control (LacZ) or rAd/U6-ARG-II IhRNA (Arg-II) for silencing. Three days posttransduction, the cells were subjected to cell counting

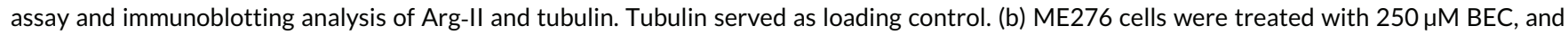
after 3 days, the cells were subjected to cell counting assay and immunoblotting analysis. (c) ME276 cells were transduced with rAd/CMV as control (Con) or rAd/CMV-ARG-II (Arg-II), or inactive mutant rAd/CMV-ARG-II-H16OF (H16OF) for overexpression. Cells were treated with or without $20 \mu \mathrm{M}$ DFMO as indicated. After 3 days of transduction, cells were subjected to cell counting assay and immunoblotting analysis of Arg-II and tubulin. endoArg-II: endogenous Arg-II; exoArg-II: overexpressed exogenous Arg-II. (d) Arginase activity assay (left bar graph) and quantification (right bar graph) of immunoblotting analysis of Arg-II protein levels shown in (c). Data are presented as mean \pm SEM. ${ }^{*} p<.05$ versus Con, ${ }^{* *} p<.01$ versus LacZ, ${ }^{* * *} p<.001$ versus Con, ${ }^{\# \#} p<.001$ versus Arg-II. rAd, recombinant adenovirus

Figure 4c. A direct hydrogen peroxide assay showed that $\mathrm{H}_{2} \mathrm{O}_{2}$ production was also reduced upon silencing (Figure 4d). Moreover, overexpression of either native or inactive Arg-II enhanced both cytoplasmic and mitochondrial $\mathrm{H}_{2} \mathrm{O}_{2}$ production (Figure S7a) with concurrent activation of p66Shc (Figure S7b), a mitochondrial redox enzyme that directly generates $\mathrm{H}_{2} \mathrm{O}_{2}$ by transferring electron from cytochrome c to oxygen (Giorgio et al., 2005). These data provide further evidence for a role of Arg-Il in promoting mitochondrial ROS production independently of its enzymatic activity.

\section{5 | Arg-II promotes melanoma cell migration and adhesion through STAT3 signaling}

To further examine whether STAT3 mediates Arg-II-promoted migration and adhesion of melanoma cells and further confirm that these are independent of the enzymatic activity of Arg-II, STAT3 inhibitor stattic was used. Treatment of the ME276 cells with stattic inhibited the increased phosphorylation of STAT3 and expression of ICAM-1 induced by the overexpression of either native or inactive Arg-II (Figure 5a,b), which was also observed in another melanoma 
(a)

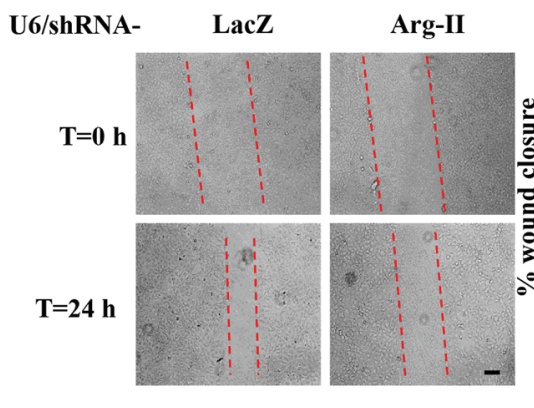

(c)

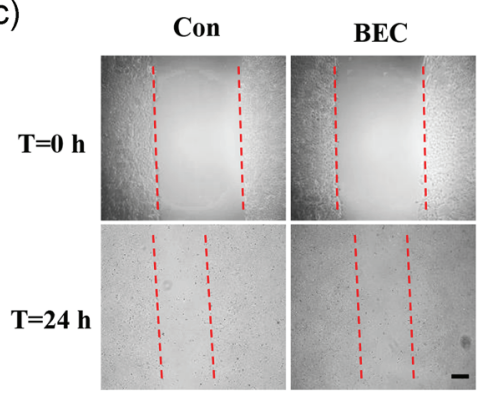

(b)

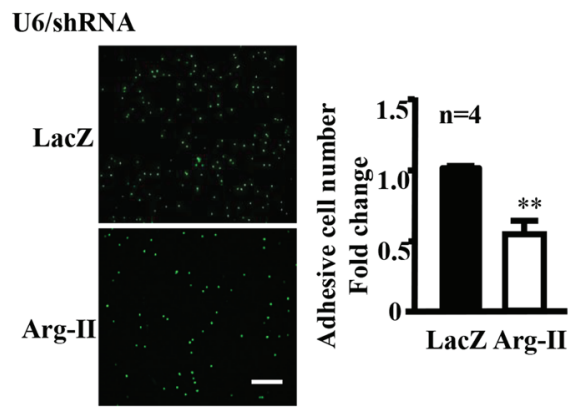

(d)
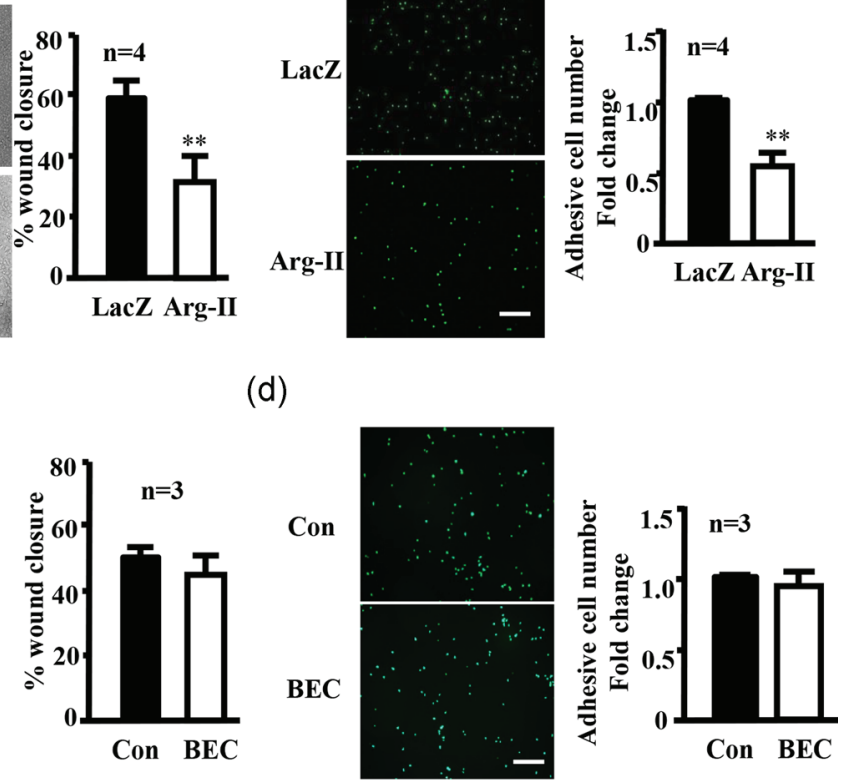

(e)
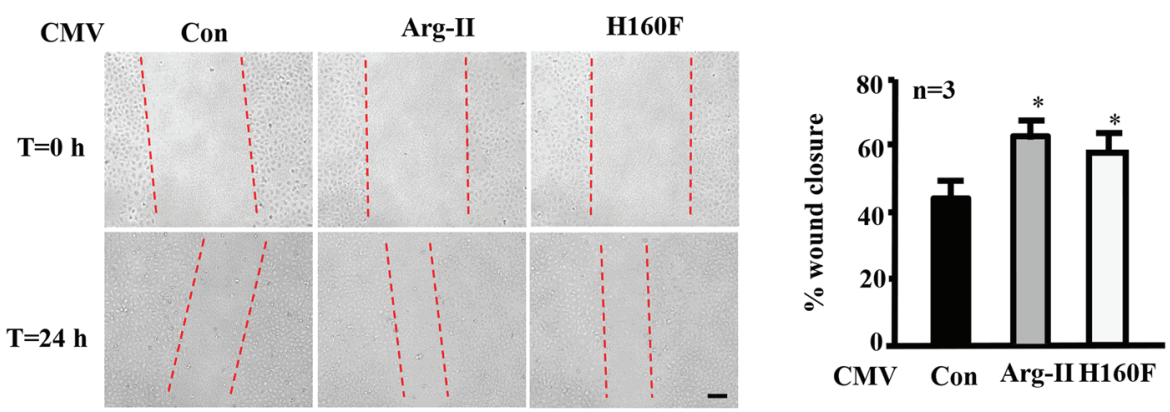

(f)
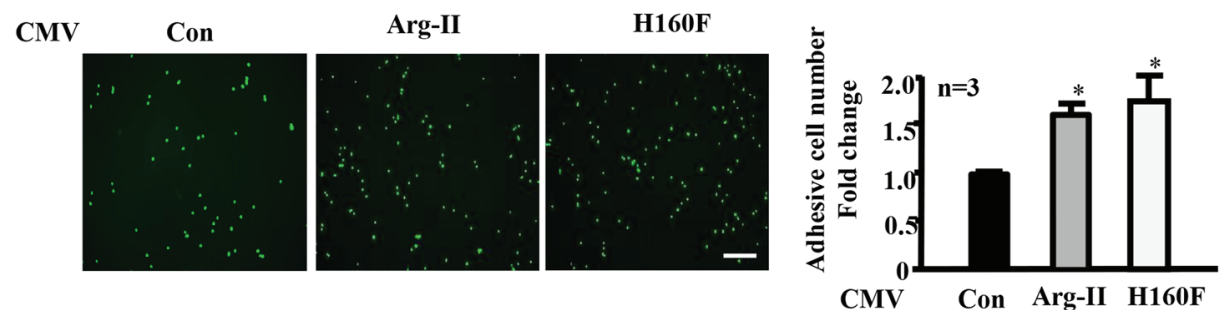

FIGURE 3 Arg-II promotes melanoma cell migration and adhesion on endothelial cells independent of its enzymatic activity. ME276 cells were ( $a$ and b) transduced with rAd/U6-LacZ ${ }^{\text {shRNA }}$ as control (LacZ) or rAd/U6-ARG-II shRNA (Arg-II) for silencing, or (c and d) treated with $250 \mu M$ BEC, or (e and f) transduced with rAd/CMV as control (Con) or rAd/CMV- ARG-II (Arg-II), or inactive mutant rAd/CMV- ARG-II -H160F (H160F) for overexpression. Three days posttransduction, the cells were subjected to (a, c, and e) wound healing assay. Images were taken at 0 and $24 \mathrm{hr}$ after scratching. Quantification of wound closure is shown in the bar graphs. (b,d, and f) Adhesion assay. Quantification of the signals is shown in the bar graphs. Data are presented as mean \pm SEM. ${ }^{*} p<.05$ versus Con, ${ }^{* *} p<.01$ versus LacZ. Scale bar $=100 \mu \mathrm{m}$. Arg-II, arginase type II; rAd, recombinant adenovirus

cell line ME275 (Figure S6b). In accordance, enhanced migration (Figure $5 c, d$ ) and adhesion ability (Figure 5 e,f) of ME276 cells overexpressing native or inactive Arg-II were dampened by the treatment with stattic, which was also observed in ME275 (Figure S4). It is to notice that inhibition of STAT3 alone also showed ability to reduce phosphorylation of STAT3 and ICAM-1 expression along with reduced adhesion and migration (Figure 5). These results indicate that the STAT3 signaling pathway contributes to Arg-II-promoted ICAM-1 expression, melanoma cell migration, and adhesion, which is independent of enzymatic activity of Arg-II.

\section{6 | Arg-II enhances STAT3 through $\mathrm{H}_{2} \mathrm{O}_{2}$ leading to enhanced melanoma cell migration and adhesion}

The fact that silencing Arg-II reduced mitochondrial ROS including $\mathrm{H}_{2} \mathrm{O}_{2}$ production in melanoma cells (Figure $4 \mathrm{c}, \mathrm{d}$ ) prompted us to 
(a)
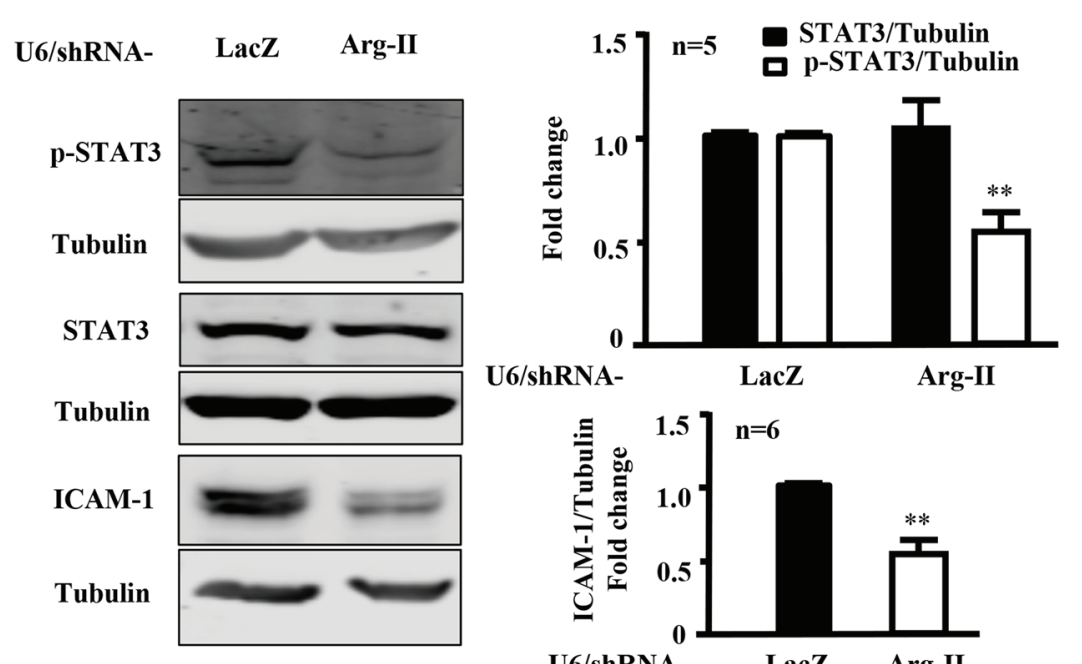

(b)
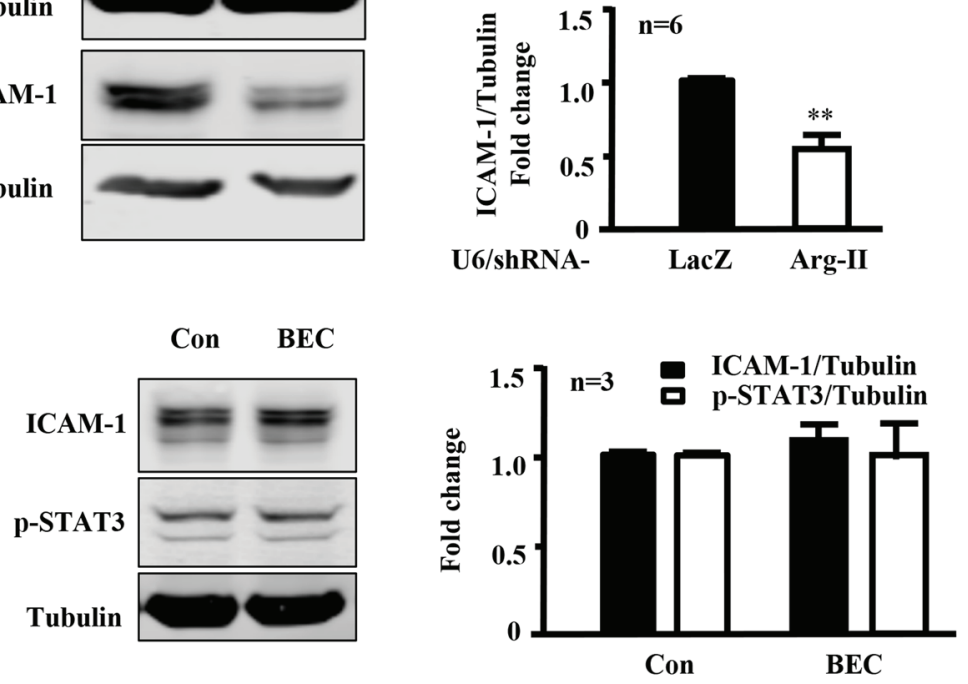

(c)

(d)
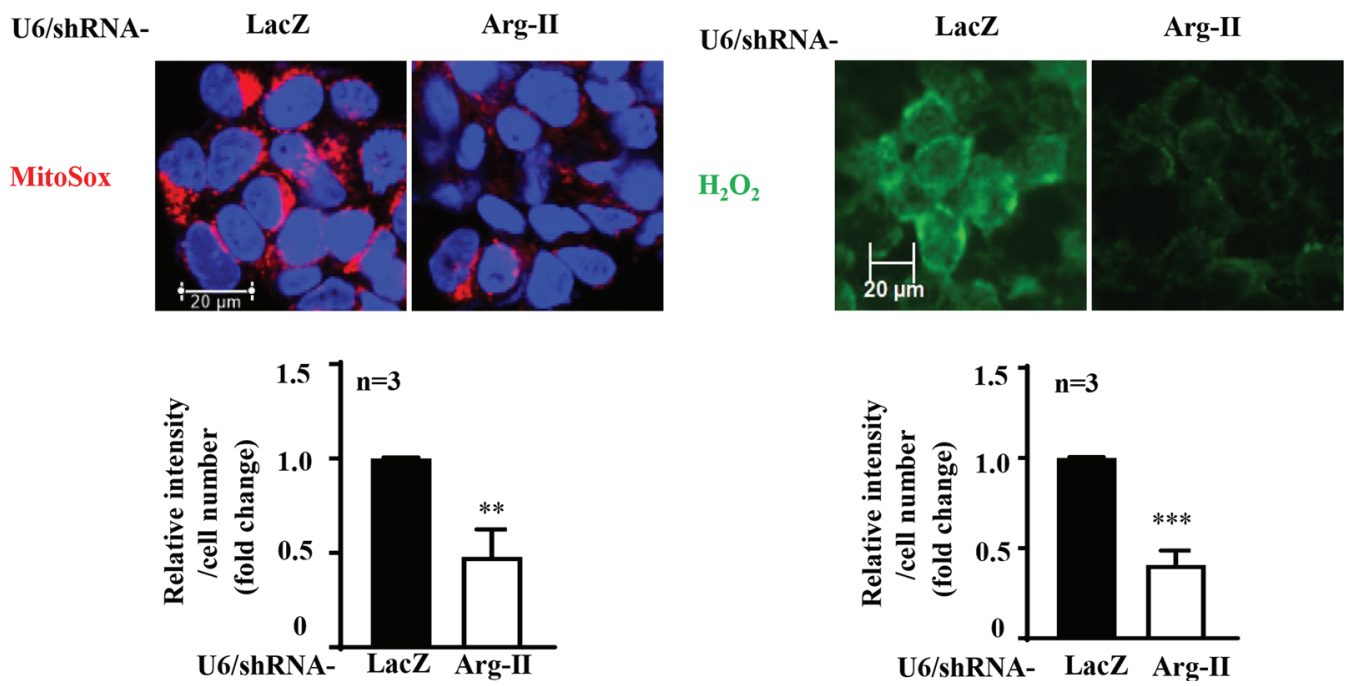

FIGURE 4 Silencing Arg-II reduces STAT3 signaling pathway and mitochondrial ROS production in melanoma cells. ME276 cells were transduced with rAd/U6-LacZ ${ }^{\text {shRNA }}$ as control (LacZ) or rAd/U6- ARG-II ${ }^{\text {shRNA }}$ (Arg-II) for silencing, or treated with $250 \mu M$ BEC. Three days posttransduction, the cells were subjected to $(a$ and $b$ ) immunoblotting analysis of p-STAT3, total STAT3, ICAM-1, and tubulin. Tubulin served as loading control. (c) Mitochondrial superoxide staining (red) followed by nuclei counter-staining with Hoechst 33342 (blue). The images were taken under confocal microscope. Quantification of the mitochondrial superoxide signals is shown in the bar graphs. (d) Hydrogen peroxide assay. The images were taken under fluorescence microscope. Data are presented as mean \pm SEM. ${ }^{* *} p<.01,{ }^{* * *} p<.001$ versus LacZ. Scale bar $=20 \mu \mathrm{m}$. Arg-II, arginase type II; rAd, recombinant adenovirus; SEM, standard error of the mean; STAT3, signal transducer and activator of transcription 3 
(a)

CMV Con Con Arg-II Arg-II H160F H160F

Stattic - $\quad+\quad-\quad+\quad+\quad-\quad+$

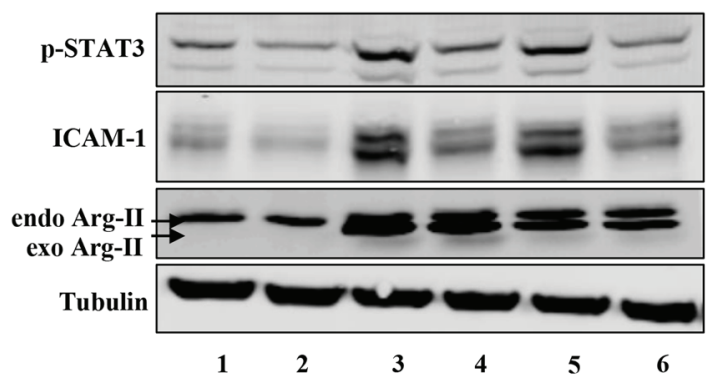

(c)

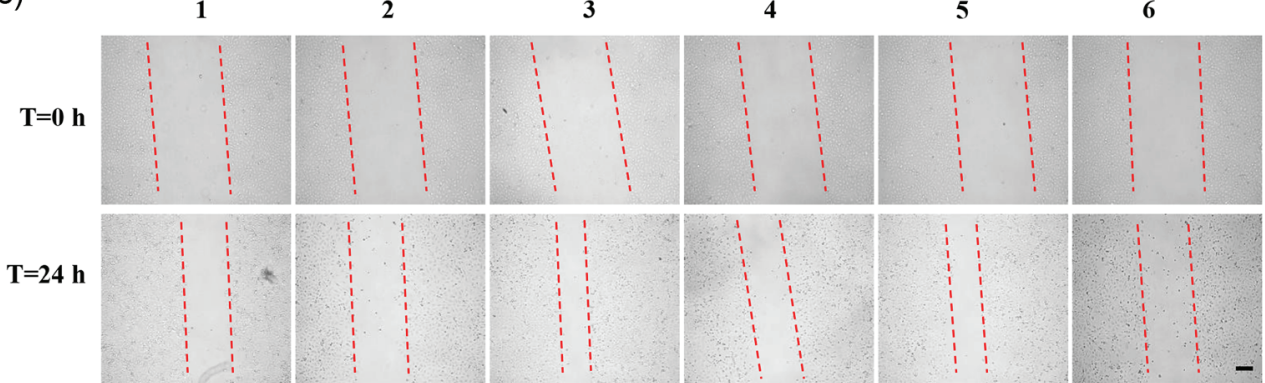

(e)

CMV

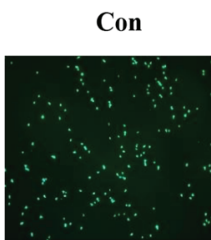

Arg-II

DMSO

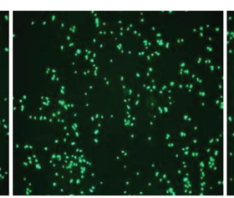

Stattic

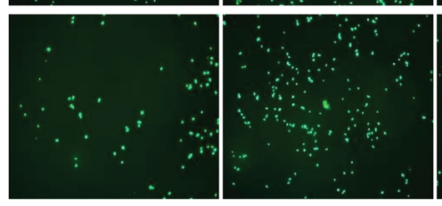

(b)

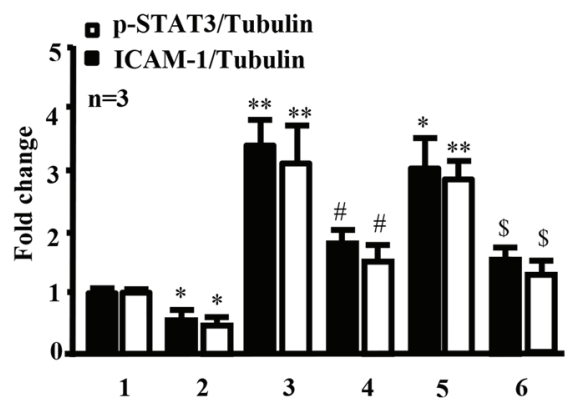

H160F

$4 \quad 5$

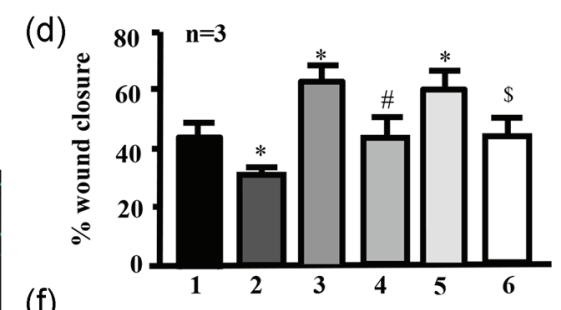

(f)
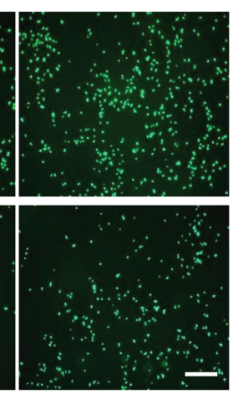

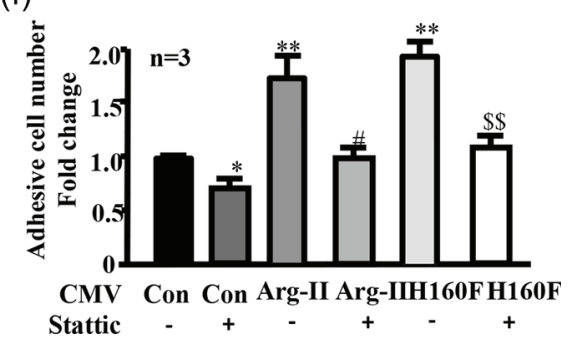

FIGURE 5 Arg-II promotes melanoma cell migration and adhesion through STAT3 signaling. ME276 cells were transduced with $\mathrm{rAd} / \mathrm{CMV}$ as control (Con), rAd/CMV-ARG-II (Arg-II), or inactive mutant rAd/CMV- ARG-II -H16OF (H160F) for overexpression. Cells were treated with $5 \mu \mathrm{M}$ STAT3 inhibitor stattic or DMSO as control. After 3 days of transduction, cells were subjected to (a) immunoblotting analysis of p-STAT3, ICAM-1, Arg-II, and tubulin. endoArg-II: endogenous Arg-II; exoArg-II: overexpressed exogenous Arg-II. (c) Wound healing assay. (e) Adhesion assay. Quantification for (a,c, and e) is shown in (b,d, and f), respectively. Data are presented as mean $\pm S E M .{ }^{*} p<.05,{ }^{* *} p<.01$ versus Con, ${ }^{\#} p<.05$ versus Arg-II, ${ }^{\$} p<.05,{ }^{\$ \$} p<.01$ versus H160F. Scale bar $=100 \mu \mathrm{m}$. Arg-II, arginase type II; DMSO, dimethyl sulfoxide; rAd, recombinant adenovirus; SEM, standard error of the mean; STAT3, signal transducer and activator of transcription 3

investigate the role of ROS in melanoma cell migration and adhesion regulated by Arg-II. As shown in Figure 6, reducing mitochondrial superoxide anion by overexpression of SOD2 in ME276 cells did not show inhibitory effect, but oppositely enhanced phosphorylation of STAT3 and ICAM-1 expression (Figure 6a) with concomitant increased migration (Figure $6 \mathrm{~b}$ ) and adhesion of melanoma cells (Figure 6c). $\mathrm{H}_{2} \mathrm{O}_{2}$, itself a product of SOD2 and generated in large amount by many human tumor cells including human melanoma cells, was reported as a central redox signaling molecule involved in tumor metastasis (Lisanti et al., 2011, Szatrowski \& Nathan, 1991). We observed that $\mathrm{H}_{2} \mathrm{O}_{2}$ enhanced phosphorylated STAT3 in ME276 cells (Figure S8). Therefore, catalase that removes $\mathrm{H}_{2} \mathrm{O}_{2}$ was overexpressed in ME276 to determine the role of $\mathrm{H}_{2} \mathrm{O}_{2}$ in Arg-II-mediated effects in melanoma cells. Immunoblotting results revealed reduced STAT3 signaling and ICAM-1 production in catalase-overexpressing cells (Figure 6a). Melanoma cell migration (Figure 6b) and adhesion onto the endothelial layer (Figure $6 \mathrm{c}$ ) were also reduced in cells overexpressing catalase, implicating the involvement of $\mathrm{H}_{2} \mathrm{O}_{2}$. 
(a)
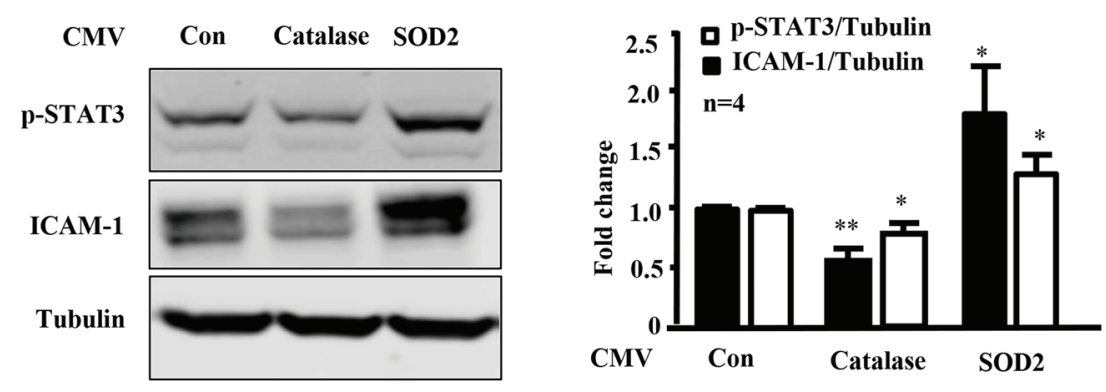

(b)
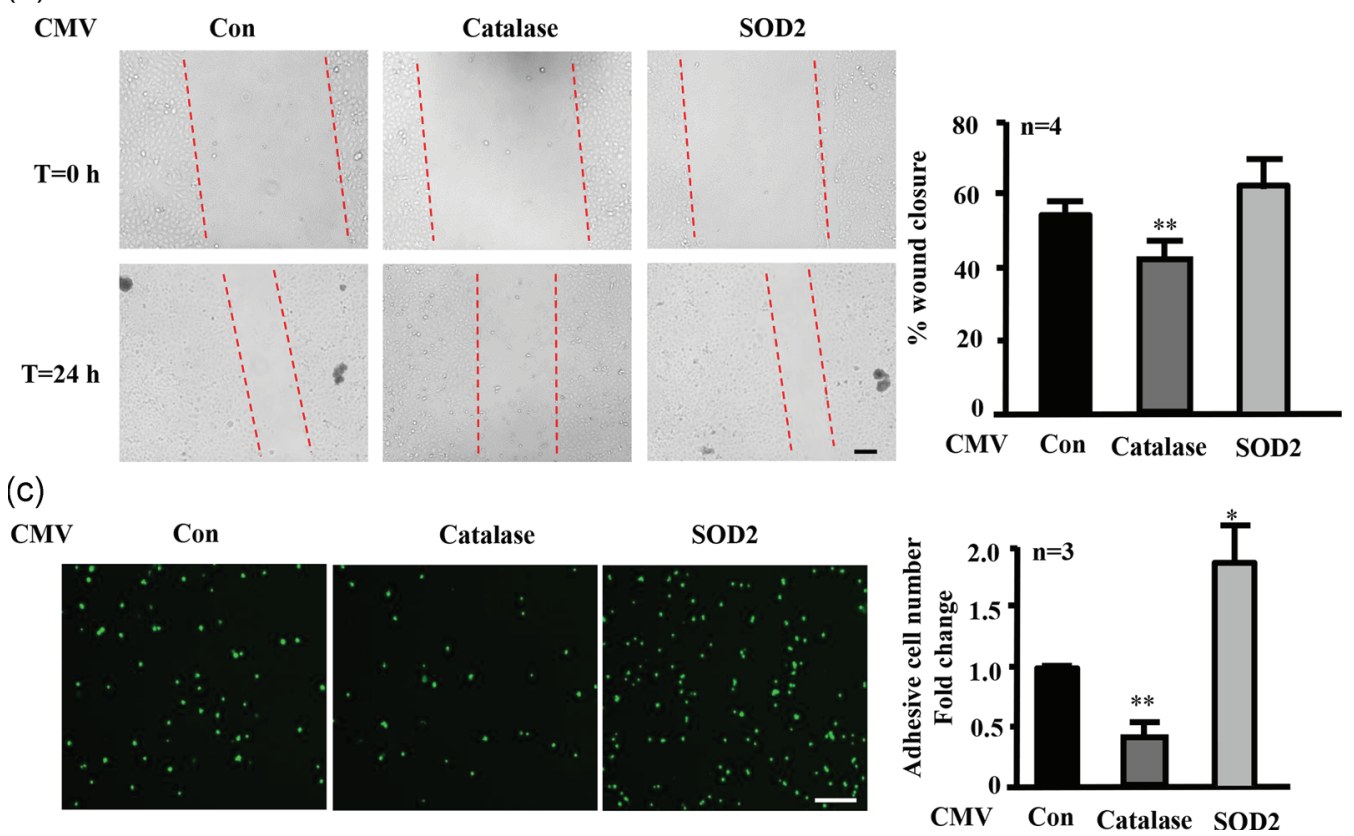

FIGURE 6 Hydrogen peroxide is involved in melanoma cell migration and adhesion. ME276 cells were transduced with $\mathrm{rAd} / \mathrm{CMV}$ as control (Con) or rAd/CMV-CAT (Catalase) or rAd/CMV-SOD2 (SOD2) for overexpression. After 3 days of transduction, cells were subjected to

(a) immunoblotting analysis of p-STAT3, ICAM-1, and tubulin. Quantification of the blots is shown in the bar graphs. (b) Wound healing assay. (c) Adhesion assay. Data are presented as mean \pm SEM. ${ }^{*} p<.05,{ }^{* *} p<.01$ versus Con. Scale bar $=100 \mu \mathrm{m}$. Arg-II, arginase type II; DMSO,

dimethyl sulfoxide; rAd, recombinant adenovirus; SEM, standard error of the mean; STAT3, signal transducer and activator of transcription 3

Next, we further examined whether Arg-II-induced STAT3 activation is mediated by $\mathrm{H}_{2} \mathrm{O}_{2}$. Figure 7 showed that phosphorylation of STAT3 and ICAM-1 expression enhanced by native or inactive Arg-II were reversed when catalase was co-overexpressed (Figure 7a,b). In line with these results, increased melanoma cell migration speed (Figure 7c,d) and adhesion onto endothelial cells (Figure 7e,f) induced by overexpression of native or inactive Arg-Il were also significantly inhibited by the coexpression of catalase. Similar results were further verified in another melanoma cell line ME275 (Figure S9). These results demonstrate that Arg-II, independently of its enzymatic activity, promotes $\mathrm{H}_{2} \mathrm{O}_{2}$ production leading to STAT3 activation, which in turn promotes melanoma cell migration and adhesion.

\section{4 | DISCUSSION}

Aberrant expression of Arg-II has been reported in various types of cancers and is closely correlated with poor outcome of patients with cancer (Bronte et al., 2005, Ino et al., 2013, Tate et al., 2008, Zaytouni et al., 2017). Moreover, the inhibition of arginase suppresses cancer cell growth and metastasis (Secondini et al., 2017, Singh et al., 2013, Tate et al., 2008, Zaytouni et al., 2017). Until now, the limited knowledge about the role of Arg-II in tumor progression has been only attributed to the suppression of cytotoxic $T$ cell due to a decrease in L-arginine availability resulting in the impaired host's antitumor immunity as well as to the enhanced cancer cell proliferation through elevated polyamine synthesis (Abete et al., 1999, Avtandilyan et al., 2018, Bronte et al., 2005, Setty et al., 2016). In the current study, we observed an association of Arg-II expression with the metastasis in patient melanoma biopsies, which is in agreement with the aforementioned studies. Though the sample size of patient melanoma tissues is small, these data nevertheless provide supporting evidence for an association of Arg-ll with the melanoma metastasis. Studies with more patient samples shall further verify this observation. Using two human melanoma cell lines as a model and arginase inhibitor and ornithine decarboxylase (ODC) inhibitor in 


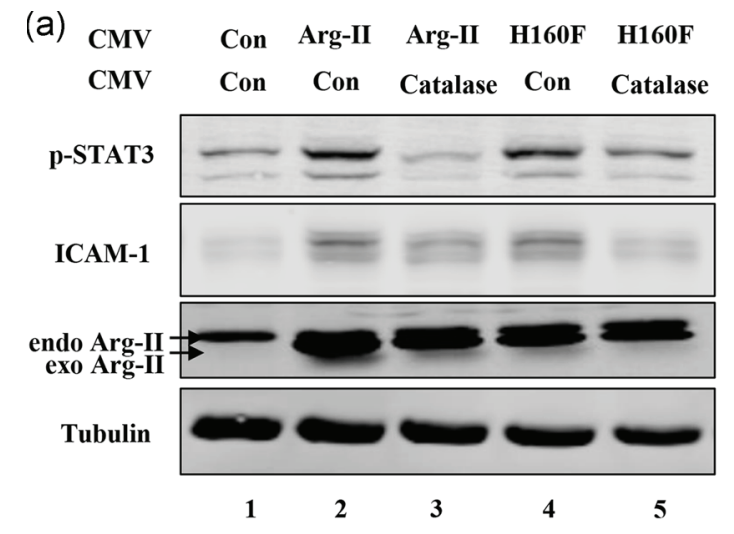

(b)

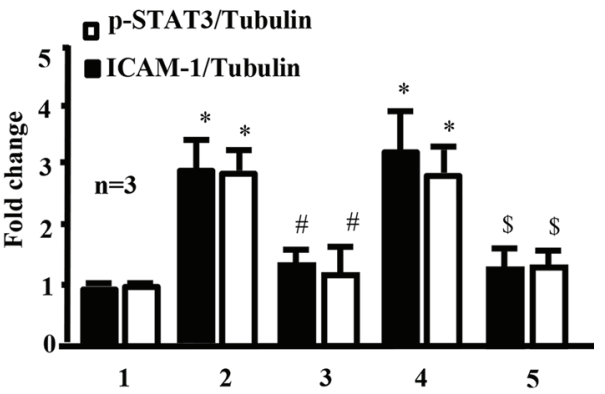

(c)

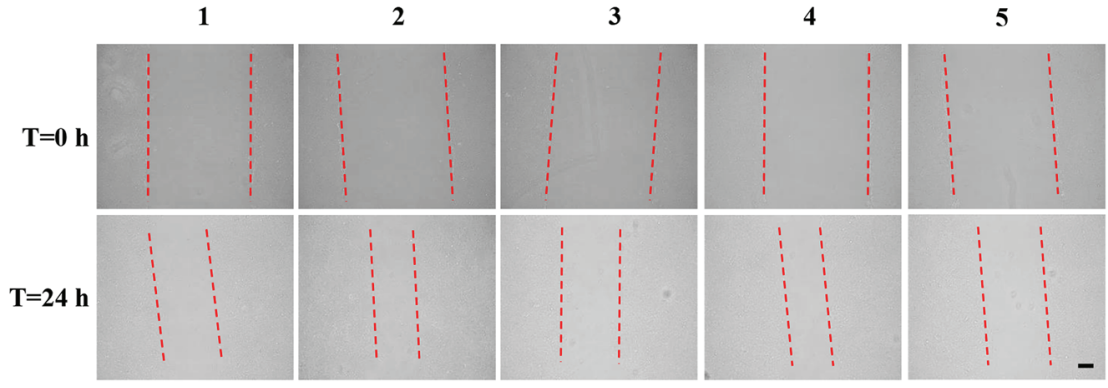

(d)

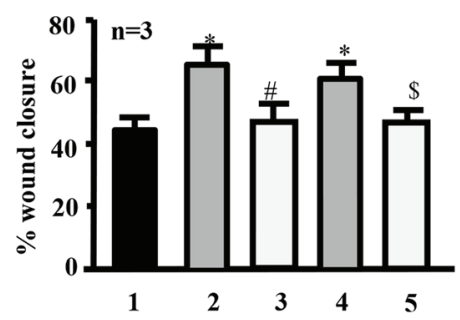

(f)

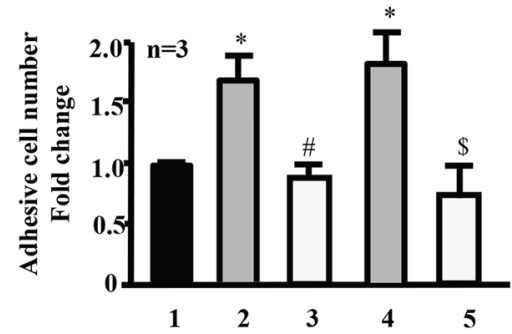

(e)

1

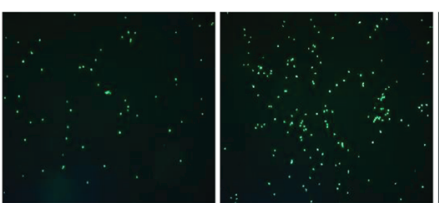

3

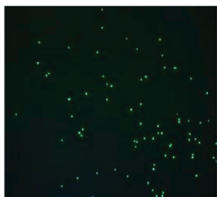

4

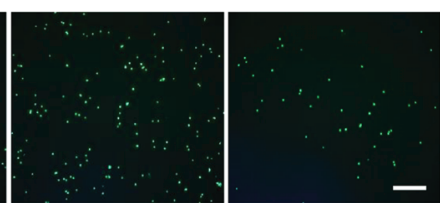

FIGURE 7 STAT3 signaling mediates melanoma cell migration and adhesion promoted by Arg-II through hydrogen peroxide production. ME276 cells were first transduced with rAd/CMV as control (Con) or rAd/CMV-CATALASE (Catalase). Twenty-four hours after the first transduction, the cells were then transduced with rAd/CMV as control (Con), rAd/CMV-ARG-II (Arg-II), or inactive mutant rAd/CMV-ARG-IIH160F (H160F). Experiments were performed 3 days post the second transduction. (a) Immunoblotting analysis of p-STAT3, ICAM-1, Arg-II, and tubulin. endoArg-II: endogenous Arg-II; exoArg-II: overexpressed exogenous Arg-II. (c) Wound healing assay. (e) Adhesion assay. Quantification for (a,c, and e) is shown in (b,d, and f), respectively. Data are presented as mean \pm SEM. ${ }^{*} p<.05$ versus Con, ${ }^{\#} p<.05$ versus Arg-II, ${ }^{\$} p<.05$ versus H16OF. Scale bar $=100 \mu \mathrm{m}$. Arg-II, arginase type II; DMSO, dimethyl sulfoxide; rAd, recombinant adenovirus; SEM, standard error of the mean; STAT3, signal transducer and activator of transcription 3

conjunction with overexpression of a native or a catalytically inactive mutant of Arg-II, we demonstrate that Arg-II promotes the melanoma cell growth dependently of its enzymatic activity, which leads to enhanced ornithine and its downstream polyamine synthesis via ODC. These data support the previous findings in other tumors (Casero \& Marton, 2007, Kingsnorth, McCann, Diekema, Ross, \& Malt, 1983, Luk, Abeloff, Griffin, \& Baylin, 1983). In addition to its essential role in cell proliferation, polyamine also enhances the invasive and metastatic capability of cancer cells (Soda, 2011). Here, we further uncovered a novel mechanism by which Arg-II promotes processes involved in metastasis including migration and adhesion independently of its enzymatic activity, which is hydrogen peroxideSTAT3 pathway, that is, Arg-ll promotes hydrogen peroxide production leading to STAT3 activation, which in turn promotes melanoma cell migration and adhesion ultimately promoting cancer metastasis. These conclusions are supported by several experimental 
evidence. First, silencing Arg-II decreases melanoma cell migration and its adhesion on endothelial cells along with reduced production mitochondrial ROS including hydrogen peroxide and STAT3 signaling in melanoma cells. Second, inhibition of STAT3 signaling dampens enhanced migration and adhesion ability of melanoma cells overexpressing Arg-II. Third, reducing hydrogen peroxide production by the coexpression of catalase reverses enhanced STAT3 signaling, melanoma cell migration activity, and adhesion onto endothelial cells induced by overexpressing Arg-II. Previous studies including our own have reported a role of an enhanced Arg-II in promoting mitochondrial ROS production in various cells including macrophages, vascular smooth muscle cells, and airway epithelial cells (Ming et al., 2012, Xiong et al., 2013, Xu et al., 2016). This finding is also confirmed in melanoma cells in the current study. The fact that enhanced STAT3 phosphorylation and ICAM-1 expression as well as the increased migration and adhesion of melanoma cells on endothelial cells upon Arg-II overexpression are not inhibited by the coexpression of SOD2 but by catalase, provides evidence that the Arg-II-promoted hydrogen peroxide production that accounts for the invasive and metastatic characteristics of melanoma cells. Our previous study in vascular smooth muscle cells demonstrated that Arg-II promotes mitochondrial hydrogen peroxide production through the activation of the mitochondrial redox protein p66Shc (Xiong et al., 2013), which may be the mechanism by which Arg-II activates hydrogen
peroxide-STAT3 leading to enhanced invasive and metastatic characteristics of melanoma cells.

One of the major findings in the study is the dual functions of Arg-II on melanoma growth and metastasis-related processes. Inactive Arg-II mutant H160F could not facilitate melanoma cell growth but promotes melanoma migration and adhesion equally as native Arg-II. Moreover, arginase inhibitor BEC effectively reduces melanoma growth but has no effect on melanoma metastasis-related processes. These data provide evidence that regulation of melanoma cell growth by Arg-II is enzymatic dependent, while the effect of Arg-II on melanoma migration and adhesion is different and independent of its activity. The Arg-II activity-independent function was also observed in our previous study. In vascular smooth muscle cells (VSMC), we for the first time uncovered a novel mechanism by which Arg-II, independently of its L-arginine ureahydrolase activity, promotes mitochondrial dysfunction leading to VSMC senescence/ apoptosis through complex positive crosstalk among S6K1-JNK, ERK, p66Shc, and p53, contributing to atherosclerotic vulnerability phenotypes in mice (Xiong et al., 2013). In HUVECs, Arg-II induces endothelial senescence and inflammation requiring enzymatic activity while enhances mTORC1/S6K1 signaling independently of its activity. It is worth noting that enzymatic activity-independent effects have been also reported for another urea cycle enzyme, argininosuccinate lyase (Erez et al., 2011), which acts as a scaffold protein.

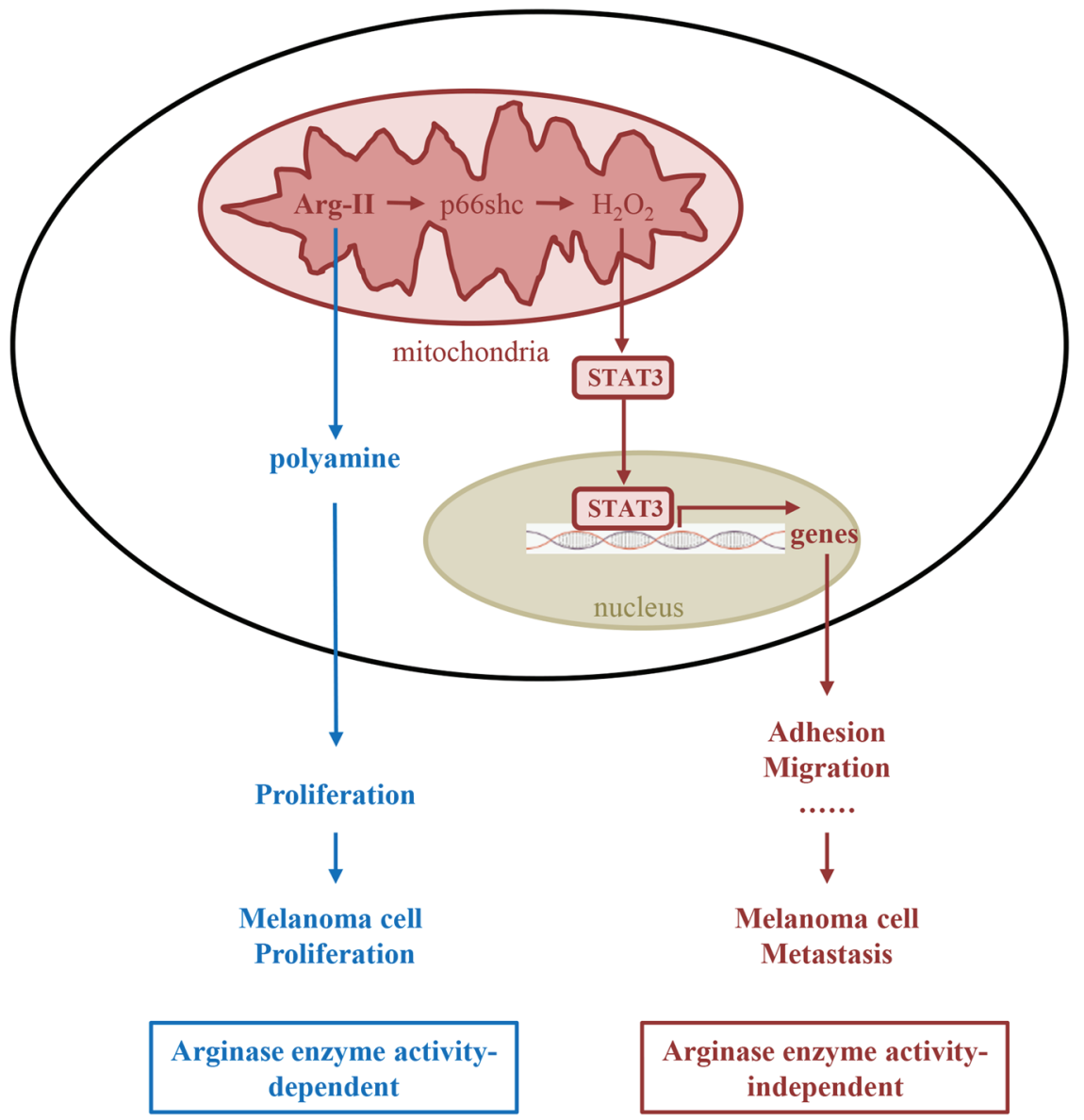

FIGURE 8 Schematic summary of the current study. Arg-II promotes melanoma progression through both activity-dependent and -independent mechanisms. While Arg-II enhances melanoma cell proliferation through polyamine dependently of its enzymatic activity, it promotes metastasis-related processes, i.e., migration and adhesion onto endothelial cell, through mitochondrial $\mathrm{H}_{2} \mathrm{O}_{2}$-STAT3 pathway independently of the enzymatic activity. Suppressing Arg-II expression rather than inhibiting its enzymatic activity may, therefore, represent a novel strategy for treatment of melanoma 
Whether this is also true for Arg-II requires further extensive investigation. The exact mechanism of this activity-independent function of Arg-II is still not completely understood. Given that the activity-independent effects of Arg-II in melanoma cells are exerted through promoting mitochondrial hydrogen peroxide production, one possible mechanism could be that Arg-II promotes mitochondrial hydrogen peroxide production at least in part through the activation of the mitochondrial redox protein p66Shc. As shown in VSMC in our previous study (Xiong et al., 2013), p66Shc is also activated by both native and inactive Arg-II in melanoma cells. How Arg-II activates p66Shc awaits further intensive investigations. This finding of the enzyme-independent role of Arg-II in melanoma provides an important hint for drug development targeting Arg-II for the treatment of melanoma or other cancers, whereby the drug suppressing Arg-II expression rather than arginase inhibitor should be developed.

Enhanced arginase (Arg-I and/or Arg-II) has been found not only in tumor cells but also in plasma of patients with cancer. On one hand, enhanced arginase in plasma reduces L-arginine availability for receptor signaling and subsequent proliferation of arginine auxotrophic T-cells leading to suppression of the T-cell-mediated antitumor immunity (Munder et al., 2006, Steggerda et al., 2017). On the other hand, enhanced arginase within tumor cells promotes cell growth through polyamine and facilitates metastasis through many features favoring metastasis such as migration and adhesion, as shown in the current study. Targeting arginase would thus be a novel promising therapeutic approach for cancers with elevated arginase in plasma and/or in the tumors. Indeed, studies demonstrate that arginase inhibition activates antitumor immunity (Miret et al., 2019) as well as suppresses cancer cell proliferation and metastasis (Secondini et al., 2017, Singh et al., 2013, Tate et al., 2008, Zaytouni et al., 2017). Given our finding in the current study that Arg-II enhances metastasis-related functions independently of its enzymatic activity, it is tempting to speculate that inhibiting Arg-Il expression would be superior to inhibiting Arg-Il activity in the treatment of cancers such as melanoma expressing high level of Arg-II.

In summary, our current study uncovers both activity-dependent and independent mechanisms of Arg-II in promoting melanoma progression. While Arg-Il enhances melanoma cell proliferation through polyamine dependently of its enzymatic activity, it promotes metastasis-related processes, that is, migration and adhesion onto endothelial cell, through mitochondrial $\mathrm{H}_{2} \mathrm{O}_{2}$-STAT3 pathway independently of the enzymatic activity (Figure 8). The highly significant association of Arg-ll with melanoma metastasis patient samples suggest that Arg-II plays an important role in the cancer metastasis. Our study implies that suppressing Arg-II expression rather than inhibiting its enzymatic activity may represent a novel strategy for the treatment of melanoma.

\section{ACKNOWLEDGMENTS}

The work was supported by the Swiss National Science Foundation (Grants 31003A_159582/1 and 31001A_179261/1) and the Swiss Heart Foundation (SHF). S. V. G. received support from the Swiss
National Science Foundation (Grants 310030_162552 and 310030_184757) and the Swiss Cancer League/Swiss Cancer Research (Grant KFS-3941-08-2016).

\section{CONFLICT OF INTERESTS}

The authors declare that there are no conflict of interests.

\section{AUTHOR CONTRIBUTIONS}

Y. Y., X.-F. M., and Z. Y. contributed to conception and design. Y. Y., K. F. B., and X. L. developed the methodology. Y. Y., D. L., Y. X., K. F. B., and X. L contributed to the acquisition of data. Y. Y., D. L., Y. X., K. F. B., X. L., S. v. G., X.-F. M., and Z. Y. were involved in the analysis and interpretation of data. Y. Y., S. v. G., X.-F. M., and Z. Y. contributed to writing, review, and/or revision of the manuscript. Y. Y., K. F. B., X. L., S. v. G., and R. E. H. proivided administrative, technical, or material support. Study supervision was done by X.-F. M. and Z. Y.

\section{ETHICS STATEMENT}

All studies using human material were conducted in accordance with the guidelines of, and approved by, the Cantonal Ethical Committees of Bern, Switzerland.

\section{ORCID}

Yi Yu (D) http://orcid.org/0000-0001-8231-6516

Yuyan Xiong (D) http://orcid.org/0000-0003-4210-7992

Xiu-Fen Ming (D) http://orcid.org/0000-0002-5848-4496

Zhihong Yang (D) http://orcid.org/0000-0002-4133-5099

\section{REFERENCES}

Abete, P., Cioppa, A., Calabrese, C., Pascucci, I., Cacciatore, F., Napoli, C., ... Rengo, F. (1999). Ischemic threshold and myocardial stunning in the aging heart. Experimental Gerontology, 34, 875-884.

Avtandilyan, N., Javrushyan, H., Petrosyan, G., \& Trchounian, A. (2018). The involvement of arginase and nitric oxide synthase in breast cancer development: Arginase and NO synthase as therapeutic targets in cancer. BioMed Research International, 2018, 8696923.

Belkhiri, A., Richards, C., Whaley, M., McQueen, S. A., \& Orr, F. W. (1997). Increased expression of activated matrix metalloproteinase- 2 by human endothelial cells after sublethal $\mathrm{H} 2 \mathrm{O} 2$ exposure. Laboratory Investigation, 77, 533-539.

Bronte, V., Kasic, T., Gri, G., Gallana, K., Borsellino, G., Marigo, I., ... Viola, A. (2005). Boosting antitumor responses of $\mathrm{T}$ lymphocytes infiltrating human prostate cancers. Journal of Experimental Medicine, 201, 1257-1268.

Caballero, O. L., Zhao, Q., Rimoldi, D., Stevenson, B. J., Svobodová, S., Devalle, S., ... Simpson, A. J. (2010). Frequent MAGE mutations in human melanoma. PLoS One, 5, e12773.

Casero, R. A., Jr., \& Marton, L. J. (2007). Targeting polyamine metabolism and function in cancer and other hyperproliferative diseases. Nature Reviews Drug Discovery, 6, 373-390.

Debidda, M., Wang, L., Zang, H., Poli, V., \& Zheng, Y. (2005). A role of STAT3 in Rho GTPase-regulated cell migration and proliferation. Journal of Biological Chemistry, 280, 17275-17285.

Dechow, T. N., Pedranzini, L., Leitch, A., Leslie, K., Gerald, W. L., Linkov, I., \& Bromberg, J. F. (2004). Requirement of matrix metalloproteinase-9 for the transformation of human mammary epithelial cells by Stat3-C. Proceedings of the National Academy of Sciences of the United States of America, 101, 10602-10607. 
Erez, A., Nagamani, S. C. S., Shchelochkov, O. A., Premkumar, M. H. Campeau, P. M., Chen, Y., ... Lee, B. (2011). Requirement of argininosuccinate lyase for systemic nitric oxide production. Nature Medicine, 17, 1619-1626.

Giorgio, M., Migliaccio, E., Orsini, F., Paolucci, D., Moroni, M., Contursi, C., ... Pelicci, P. G. (2005). Electron transfer between cytochrome c and p66Shc generates reactive oxygen species that trigger mitochondrial apoptosis. Cell, 122, 221-233.

Gokmen, S. S., Aygit, A. C., Ayhan, M. S., Yorulmaz, F., \& Gulen, S. (2001). Significance of arginase and ornithine in malignant tumors of the human skin. Journal of Laboratory and Clinical Medicine, 137, 340-344.

Guo, N., Shen, G., Zhang, Y., Moustafa, A. A., Ge, D., \& You, Z. (2019). Interleukin-17 promotes migration and invasion of human cancer cells through upregulation of MTA1 expression. Frontiers in Oncology, 9, 546.

Ino, Y., Yamazaki-Itoh, R., Oguro, S., Shimada, K., Kosuge, T., Zavada, J., ... Hiraoka, N. (2013). Arginase II expressed in cancer-associated fibroblasts indicates tissue hypoxia and predicts poor outcome in patients with pancreatic cancer. PLoS One, 8, e55146.

Kamran, M. Z., Patil, P., \& Gude, R. P. (2013). Role of STAT3 in cancer metastasis and translational advances. BioMed Research International, 2013, 421821.

Kingsnorth, A. N., McCann, P. P., Diekema, K. A., Ross, J. S., \& Malt, R. A. (1983). Effects of alpha-difluoromethylornithine on the growth of experimental Wilms' tumor and renal adenocarcinoma. Cancer Research, 43, 4031-4034.

Kusano, T., Berberich, T., Tateda, C., \& Takahashi, Y. (2008). Polyamines: Essential factors for growth and survival. Planta, 228, 367-381.

Lisanti, M. P., Martinez-Outschoorn, U. E., Lin, Z., Pavlides, S., WhitakerMenezes, D., Pestell, R. G., ... Sotgia, F. (2011). Hydrogen peroxide fuels aging, inflammation, cancer metabolism and metastasis: The seed and soil also needs "fertilizer". Cell Cycle, 10, 2440-2449.

Luk, G. D., Abeloff, M. D., Griffin, C. A., \& Baylin, S. B. (1983). Successful treatment with DL-alpha-difluoromethylornithine in established human small cell variant lung carcinoma implants in athymic mice. Cancer Research, 43, 4239-4243.

Ming, X. F., Barandier, C., Viswambharan, H., Kwak, B. R., Mach, F., Mazzolai, L., ... Yang, Z. (2004). Thrombin stimulates human endothelial arginase enzymatic activity via RhoA/ROCK pathway: Implications for atherosclerotic endothelial dysfunction. Circulation, 110, 3708-3714.

Ming, X. F., Rajapakse, A. G., Yepuri, G., Xiong, Y., Carvas, J. M., Ruffieux, J., ... Yang, Z. (2012). Arginase II promotes macrophage inflammatory responses through mitochondrial reactive oxygen species, contributing to insulin resistance and atherogenesis. Journal of the American Heart Association, 1, e000992.

Ming, X. F., Viswambharan, H., Barandier, C., Ruffieux, J., Kaibuchi, K., Rusconi, S., \& Yang, Z. (2002). Rho GTPase/Rho kinase negatively regulates endothelial nitric oxide synthase phosphorylation through the inhibition of protein kinase B/Akt in human endothelial cells. Molecular and Cellular Biology, 22, 8467-8477.

Miret, J. J., Kirschmeier, P., Koyama, S., Zhu, M., Li, Y. Y., Naito, Y., ... Akbay, E. A. (2019). Suppression of Myeloid Cell Arginase Activity leads to Therapeutic Response in a NSCLC Mouse Model by Activating Anti-Tumor Immunity. Journal for Immunotherapy of Cancer, 7, 32

Munder, M., Schneider, H., Luckner, C., Giese, T., Langhans, C. D., Fuentes, J. M., ... Ho, A. D. (2006). Suppression of T-cell functions by human granulocyte arginase. Blood, 108, 1627-1634.

Niu, G., Wright, K. L., Ma, Y., Wright, G. M., Huang, M., Irby, R., ... Yu, H. (2005). Role of Stat3 in regulating p53 expression and function. Molecular and Cellular Biology, 25, 7432-7440.

Porembska, Z., Luboinski, G., Chrzanowska, A., Mielczarek, M., Magnuska, J., \& Baranczyk-Kuzma, A. (2003). Arginase in patients with breast cancer. Clinica Chimica Acta, 328, 105-111.
Porembska, Z., Zabek, J., Grabon, W., Rahden-Staron, I., \& BaranczykKuzma, A. (2001). Arginase isoforms in human colorectal cancer. Clinica Chimica Acta, 305, 157-165.

Rotondo, R., Mastracci, L., Piazza, T., Barisione, G., Fabbi, M., Cassanello, M., ... Frumento, G. (2008). Arginase 2 is expressed by human lung cancer, but it neither induces immune suppression, nor affects disease progression. International Journal of Cancer, 123, 1108-1116.

Schaefer, L. K., Ren, Z., Fuller, G. N., \& Schaefer, T. S. (2002). Constitutive activation of Stat3alpha in brain tumors: Localization to tumor endothelial cells and activation by the endothelial tyrosine kinase receptor (VEGFR-2). Oncogene, 21, 2058-2065.

Secondini, C., Coquoz, O., Spagnuolo, L., Spinetti, T., Peyvandi, S., Ciarloni, L., ... Ruegg, C. (2017). Arginase inhibition suppresses lung metastasis in the $4 \mathrm{~T} 1$ breast cancer model independently of the immunomodulatory and anti-metastatic effects of VEGFR-2 blockade. Oncoimmunology, 6, e1316437.

Setty, B. A., Jin, Y., Houghton, P. J., Yeager, N. D., Gross, T. G., \& Nelin, L. D. (2016). Hypoxic proliferation of osteosarcoma cells depends on arginase II. Cellular Physiology and Biochemistry, 39, 802-813.

Singh, R., Avliyakulov, N. K., Braga, M., Haykinson, M. J., Martinez, L, Singh, V., ... Pervin, S. (2013). Proteomic identification of mitochondrial targets of arginase in human breast cancer. PLoS One, 8, e79242.

Siveen, K. S., Sikka, S., Surana, R., Dai, X., Zhang, J., Kumar, A. P., ... Bishayee, A. (2014). Targeting the STAT3 signaling pathway in cancer: Role of synthetic and natural inhibitors. Biochimica et Biophysica Acta, 1845, 136-154.

Soda, K. (2011). The mechanisms by which polyamines accelerate tumor spread. Journal of Experimental \& Clinical Cancer Research, 30, 95.

Steggerda, S. M., Bennett, M. K., Chen, J., Emberley, E., Huang, T., Janes, J. R., ... Gross, M. I. (2017). Inhibition of arginase by CB-1158 blocks myeloid cell-mediated immune suppression in the tumor microenvironment. Journal for ImmunoTherapy of Cancer, 5, 101.

Szatrowski, T. P., \& Nathan, C. F. (1991). Production of large amounts of hydrogen peroxide by human tumor cells. Cancer Research, 51, 794-798.

Tang, D. G., \& Honn, K. V. (1994). Adhesion molecules and tumor metastasis: An update. Invasion and Metastasis, 14, 109-122.

Tate, D. J., Jr., Vonderhaar, D. J., Caldas, Y. A., Metoyer, T., Patterson, J. R. T., Aviles, D. H., \& Zea, A. H. (2008). Effect of arginase II on L-arginine depletion and cell growth in murine cell lines of renal cell carcinoma. Journal of Hematology \& Oncology, 1, 14.

Timosenko, E., Hadjinicolaou, A. V., \& Cerundolo, V. (2017). Modulation of cancer-specific immune responses by amino acid degrading enzymes. Immunotherapy, 9, 83-97.

Wirtz, D., Konstantopoulos, K., \& Searson, P. C. (2011). The physics of cancer: The role of physical interactions and mechanical forces in metastasis. Nature Reviews Cancer, 11, 512-522.

Xie, T. X., Wei, D., Liu, M., Gao, A. C., Ali-Osman, F., Sawaya, R., \& Huang, S. (2004). Stat3 activation regulates the expression of matrix metalloproteinase- 2 and tumor invasion and metastasis. Oncogene, 23, 3550-3560.

Xiong, Y., Yu, Y., Montani, J. P., Yang, Z., \& Ming, X. F. (2013). Arginase-II induces vascular smooth muscle cell senescence and apoptosis through p66Shc and p53 independently of its I-arginine ureahydrolase activity: Implications for atherosclerotic plaque vulnerability. Journal of the American Heart Association, 2, e000096.

Xu, W., Ghosh, S., Comhair, S. A., Asosingh, K., Janocha, A. J., Mavrakis, D. A., ... Erzurum, S. C. (2016). Increased mitochondrial arginine metabolism supports bioenergetics in asthma. Journal of Clinical Investigation, 126, 2465-2481.

Yepuri, G., Velagapudi, S., Xiong, Y. Y., Rajapakse, A. G., Montani, J. P., Ming, X. F., \& Yang, Z. H. (2012). Positive crosstalk between arginase-II and S6K1 in vascular endothelial inflammation and aging. Aging cell, 11, 1005-1016. 
Zaytouni, T., Tsai, P. Y., Hitchcock, D. S., DuBois, C. D., Freinkman, E., Lin, L., ... Kalaany, N. Y. (2017). Critical role for arginase 2 in obesityassociated pancreatic cancer. Nature Communications, 8, 242.

\section{SUPPORTING INFORMATION}

Additional supporting information may be found online in the Supporting Information section.
How to cite this article: Yu Y, Ladeiras D, Xiong Y, et al. Arginase-Il promotes melanoma migration and adhesion through enhancing hydrogen peroxide production and STAT3 signaling. J Cell Physiol. 2020;1-15.

https://doi.org/10.1002/jcp.29814 